\title{
INSTITUCIONALIZACIÓN Y CONSUMO CULTURAL: CONTINUIDADES Y TRANSFORMACIONES DE LAS LÓGICAS DE ACCESO A LAS ARTES EN CHILE (2003-2017)*
}

\author{
INSTITUTIONALIZATION AND CULTURAL CONSUMPTION: \\ CONTINUITIES AND TRANSFORMATIONS OF THE ACCESS \\ TO THE ARTS LOGICS IN CHILE (2003-2017)
}

\section{TOMÁS PETERS ${ }^{\star *}$}

Resumen: Este artículo describe y analiza las trayectorias del consumo cultural de las/os chilenas/os desde la creación del Consejo Nacional de la Cultura y las Artes en 2003 hasta 2017. Por una parte, presenta una breve revisión histórica de las políticas culturales en Chile y establece los principios que definen el acceso cultural como una estrategia de política pública. Por otra, y usando las cuatro encuestas implementadas por el CNCA, el artículo describe las principales trayectorias y mutaciones del acceso a las artes en Chile. En tercer lugar, usando un análisis de correspondencias múltiples de la Encuesta de Participación Cultural 2017, este artículo presenta un análisis de cluster con los actuales perfiles del consumo cultural de las/os chilenas/os. Este análisis identificó cuatro perfiles: alto acceso (15\%), acceso medio (20\%), acceso bajo (27\%) y acceso muy bajo (37\%). Al igual que en estudios previos desarrollados en el campo, no hay evidencia de cambios estructurales en la estratificación del consumo cultural en Chile en la última década.

Palabras Clave: Consumo Cultural, políticas culturales, desigualdad, digitalización, Chile.

AвsтRACT: This paper describes and analyses the cultural consumption trajectories of Chilean people from the creation of the National Council of Culture and the Arts (NCCA) in 2003, until 2017. On the one hand, it shows a brief historical revision of Chile's cultural policies and defines the principles that organize the cultural access as a strategic public policy. On the other, using the four surveys implemented in the NCCA, it presents the main trajectories and mutations of the cultural access in Chile. Thirdly,

\footnotetext{
* Este artículo es resultado del Proyecto Fondecyt de postdoctorado № 3180346, "Participación y consumo cultural en Chile en el siglo XXI: prácticas emergentes, nuevas desigualdades y redefiniciones teóricoculturales".

** Doctor en Estudios Culturales. Académico e investigador del Instituto de la Comunicación e Imagen, Universidad de Chile, Santiago, Chile. Correo electrónico: tpeters@uchile.cl. Orcid: https:// orcid.org/0000-0002-0765-917X
} 
using a multiple correspondence analysis of the 2017 Survey of Cultural Participation in Chile, this paper describes a cluster analysis with the current profiles of Chileans cultural consumers. The analysis identifies four profiles: high access (15\%), middle access (20\%), low access (27\%) and very low access (37\%). According to previous research developed in the field, there is no evidence of structural changes in the stratification of cultural consumption in Chile in the last decade.

Keywords: Cultural consumption, Cultural Policies, Inequality, Digitalization, Chile.

Recibido: 21.05.2020. Aceptado: 02.03.2021

\section{INTRODUCCIÓN}

$\mathrm{D}$ ESDE LA CREACIÓn DEL Consejo Nacional de la Cultura y las Artes de Chile (CNCA) en 2003, se ha implementado una serie de planes y programas orientados a fomentar el desarrollo cultural y patrimonial del país. Reconociendo el principio del "derecho al acceso cultural" (Odello, 2011), el Estado de Chile ha dedicado especial atención política y financiera a facilitar y promover el consumo y participación cultural en la sociedad chilena (CNCA, 2014). Con el objetivo de medir, sistematizar y establecer una lógica investigativa histórica de esa función, desde 2004 el CNCA ha realizado cuatro encuestas nacionales de consumo y participación cultural comparables entre sí (2005, 2009, 2012 y 2017). La tendencia estadística demuestra que, durante estos quince años, se vivió un crecimiento exponencial en el consumo y participación cultural de los chilenos entre los primeros años de la institucionalidad cultural (2005 y 2011), pero, desde la medición de 2012 y especialmente la de 2017, se ha evidenciado una caída significativa en los accesos a presentaciones de teatro y danza, exposiciones de artes visuales, prácticas de lectura y asistencia a museos y bibliotecas. A pesar de la inversión pública e institucionalización cultural de los últimos años -en febrero de 2018 el CNCA es reemplazado por un Ministerio de las Culturas, las Artes y el Patrimonio-, las políticas culturales se han visto crecientemente desafiadas a cumplir los propósitos de ampliación de los accesos y consumos culturales de la población nacional. En un contexto de aceleración de las temporalidades y complejización en las dinámicas socioculturales contemporáneas -caracterizadas por la expansión de plataformas digitales, precarización de las condiciones sociales estructurales (trabajo, previsión social, salud, vivienda, transporte, etcétera) y las nuevas tendencias de las industrias culturales y creativas (Martineau, 2017; Miller, 2018)-, las/os chilenas/os han experimentado nuevas lógicas de participación cultural, 
social y política. Este artículo estudia y describe las trayectorias del acceso cultural en Chile en los últimos quince años. En una primera parte, realiza una breve revisión histórica de los principios políticos que han orientado el acceso cultural como una línea estratégica de política pública. En una segunda sección, se exponen las principales oscilaciones y trayectorias del acceso cultural en Chile a partir de la revisión de las cuatro encuestas antes reseñadas. En tercer lugar, y a través de un análisis de correspondencias múltiples elaborado a partir de la IV Encuesta de Participación Cultural en Chile (2017), este artículo desarrolla un análisis de cluster con los actuales perfiles de consumidores culturales de los chilenos. Los resultados indican que el consumo cultural mantiene una fuerte correlación con ingreso (capital económico) y escolaridad (capital cultural). Al mismo tiempo, el acceso a internet no solo ha logrado una penetración importante en la vida de las/ os chilenas/os, sino que también ha servido como un proveedor importante de accesos culturales como lectura, video, música y cine. Junto con constatar una disminución general de los consumos culturales tradicionales (acceso a teatro, artes visuales, museos, bibliotecas, danza), los resultados muestran que, en la población más joven, se genera una concentración de los consumos culturales en festivales de música popular y acceso a salas de cine. Finalmente, este artículo elabora algunas hipótesis de lectura sobre las tendencias y problemas aquí descritos, así como también plantea desafíos tanto para la política cultural como para la sociología de la cultura.

\section{POLÍTICAS, INSTITUCIONALIZACIÓN Y CONSUMO CULTURAL EN CHILE}

Al retorno de la democracia en 1990, en Chile se gestaron variados procesos de institucionalización política, social y cultural (Catalán y Sunkel, 1990). Uno de esos hitos fue la creación de la "División de Cultura" del Ministerio de Educación, la cual definió los primeros lineamientos estratégicos del fomento a la producción artística y el acceso a la cultura (Carrasco y Negrón, 2006). En un contexto de apertura política y comercial, la producción cultural vivió una nueva etapa marcada por la expansión de la oferta y demanda de bienes y servicios artísticos (Carrasco y Negrón, 2005), así como también de un nuevo uso del espacio público (Pinochet, 2016). A finales de la década de 1990, se gestaron nuevos canales televisivos y estaciones radiales, se formaron productoras de grandes eventos (conciertos de gran público, festivales callejeros), existió un crecimiento editorial renova- 
do, se produjo una inversión millonaria de sellos musicales internacionales en la escena local, la industria cinematográfica nacional se inscribió en el circuito global, etcétera (Brunner, 2005; Ubilla, 2010). Estos procesos de masificación del consumo cultural global generaron nuevos desafíos tanto políticos como teóricos.

Luego de variadas comisiones y diagnósticos sobre la necesidad de contar con un ente público de mayor complejidad organizacional en materia cultural, el año 2003 se dio paso a la creación del "Consejo Nacional de la Cultura y las Artes" de Chile. Este segundo hito de institucionalización cultural significó un avance clave en materia de fomento cultural en la posdictadura (Carrasco y Negrón, 2006; Antoine, 2009; Muñoz, 2011; Palominos, 2018). Con el surgimiento de estas primeras políticas culturales "oficiales" del Estado de Chile, se crearon planes, programas e inversiones en materia cultural inéditos para el país: se fundaron nuevos centros culturales, se crearon consejos regionales de cultura a lo largo del país, se ampliaron los fondos concursables a diversas áreas del mundo artístico, se elaboraron políticas específicas para el fomento de las industrias culturales y creativas, se gestaron programas de educación y mediación artística en los espacios culturales del país, etcétera. Varios han sido los autores que, desde la sociología de la cultura, han demostrado la complejidad sistémica, cultural y política de este proceso histórico (Brunner, 1990 y 2005; PNUD, 2002; Garretón, 2008; De Cea, 2010 y 2017; Güell, 2012).

Luego de 13 años de historia del Consejo y con la emergencia de nuevas demandas sociales -gratuidad en educación, protección social, exigibilidad/matriz de derechos, entre otras luchas sociales emergentes-, desde el año 2013 surgió la necesidad de legislar sobre la conformación de una instancia de mayor complejidad organizacional que permitiera, por una parte, lograr una institucionalidad cultural y, por otra, reducir la dispersión y duplicidad de roles, funciones y competencias que, desde la recuperación democrática en 1990, existía en el sector público dedicado a temáticas culturales. En marzo de 2018, y luego de variados procesos de participación y discusiones legislativas durante el año 2017, se dio paso a una tercera y nueva etapa de institucionalización cultural con la creación del "Ministerio de las Culturas, las Artes y el Patrimonio". Este nuevo capítulo de la institucionalidad cultural exige investigaciones que establezcan "líneas analíticas" sobre lo hecho históricamente en materia de acceso a las artes en Chile, así como también ayuden a definir marcos de análisis para el futuro de las políticas culturales. 


\section{TRAYECTORIA DE LA PARTICIPACIÓN CULTURAL}

EN CHILE ENTRE 2003-2017

Una de las principales políticas culturales implementadas en Chile ha sido incentivar el acceso y participación cultural de sus ciudadanos. A inicios de la década del 2000, el diagnóstico general señalaba, por una parte, que las instituciones políticas estaban en "deuda" con la cultura, lo que generó la necesidad de coordinar, apoyar y promover la producción y condiciones generales de existencia del campo cultural local (Navarro, 2006). Por otra parte, la apuesta estaba dada en que la sociedad chilena demostraba una alta demanda de actividades y bienes artísticos que no estaba siendo satisfecha (Rampaphorn, 2008). Debido a la falta de espacios culturales (teatros, salas de concierto, centros culturales, museos) a inicios del 2000, resultaba necesario construirlos, para así dar cabida no solo a las manifestaciones artísticas en proceso de producción y exhibición, sino también -y sobre todo- para permitir el acceso a la cultura a la sociedad en su conjunto. Estas directrices, emanadas de comisiones de expertos (De Cea, 2010) y, posteriormente, en las Políticas Culturales quinquenales (2005-2010, 2011-2016 y 2017-2022), dieron paso a un crecimiento importante del presupuesto público, así como también una inversión sostenida en infraestructura cultural (CNCA, 2017). Al mismo tiempo, se crearon Consejos específicos para los principales dominios artísticos (tales como el de la industria audiovisual, del libro y la lectura y de la música nacional) y políticas específicas de fomento para cada sector. En su conjunto, se edificó una institucionalidad cultural que ha buscado construir un escenario propicio para dar respuesta a las demandas y necesidades culturales de las/os chilenas/os. Luego de quince años de intervención pública, resulta necesario estudiar y analizar las trayectorias del acceso cultural en Chile y establecer lecturas críticas sobre el fenómeno.

Con el objetivo de hacer un seguimiento y análisis de las lógicas, dinámicas y formas de consumo cultural de las/os chilenas/os, desde el año 2005 se han realizado cuatro encuestas de participación y consumo cultural: "Encuesta de Consumo Cultural 2005", "Segunda Encuesta Nacional de Participación y Consumo Cultural 2009", "Encuesta Nacional de Participación y Consumo Cultural 2012" y "Encuesta Nacional de Participación Cultural 2017". Pensadas como insumos analíticos, estas encuestas han establecido un criterio de comparabilidad en las variables principales de acceso y frecuencia, así como también de dimensiones específicas por cada dominio 
artístico. Todas tienen representatividad a nivel país, un nivel de confianza de $95 \%$ y error muestral nacional en promedio de $1,5 \%$. Si bien cada una de ellas ha tenido enfoques particulares según cada administración política -y se establecieron registros analíticos de mayor o menor profundidad-, todas han mantenido una coherencia lógica que permite establecer líneas históricas de análisis. El criterio compartido de todas las encuestas es la variable acceso, medida por haber asistido a una exposición de artes visuales, presentación de danza y/o teatro, visita a museo y/o biblioteca, ver una película en el cine y haber leído un libro, al menos una vez, en los últimos doce meses desde la realización de la pregunta. Esto ha sido un principio utilizado por todas las encuestas hasta la fecha.

La tendencia estadística demuestra que, durante estos quince años, se evidencia un crecimiento exponencial en el consumo cultural de las/os chilenas/os entre los primeros años de la institucionalidad cultural (2005 y 2011), pero, desde la medición de 2012 y especialmente la de 2017, se ha producido una caída significativa en los accesos a las artes consideradas como tradicionales. En el Gráfico No 1 se evidencia esta tendencia. En la medición de 2005 se observa la concentración del porcentaje de acceso de gran parte de los dominios artísticos entre un $15 \%$ y $25 \%$. En el caso de acceso al cine la cifra es significativamente diferente, con cerca de un $35 \%$. Al avanzar en la medición de 2009, estas cifras no se modificaron sustantivamente -aunque en algunos casos se observó una leve disminución, como en bibliotecas, teatro y artes visuales-, salvo en el caso de lectura de libros (desde 2005 a 2009, esta práctica experimentó un crecimiento importante, desde un $22,6 \%$ a un $41,4 \%$ en cuatro años). Algo similar ocurrió con el caso de danza: en 2005 el valor fue de 14,7\% y en 2009 de 23,5\%. En el caso de cine y museos, el porcentaje se mantuvo relativamente estable con respecto a la medición anterior.

Entre 2009 y 2012, se observan cambios importantes en los accesos a los diversos dominios artísticos. En el caso de lectura, se observa un crecimiento sustantivo: de $41,4 \%$ a un $47 \%$. En el caso del acceso al cine el ascenso es sustantivo: de un $34,9 \%$ en 2009 a un $45,2 \%$ en 2012 . También es posible identificar un crecimiento leve en el acceso a museos y artes visuales: de $20,8 \%$ a $23,6 \%$ en el primer caso y de un $22,2 \%$ a un $24,9 \%$ en el segundo. Si bien estos accesos manifestaron un aumento notorio, no ocurrió lo mismo con danza (de $23,5 \%$ en 2009 a 22,9\% en 2012), teatro (de 18,6\% en 2009 a $17,8 \%$ en 2012) y acceso a bibliotecas (de $21,6 \%$ en 2009 a $18,2 \%$ en 2012 ). 


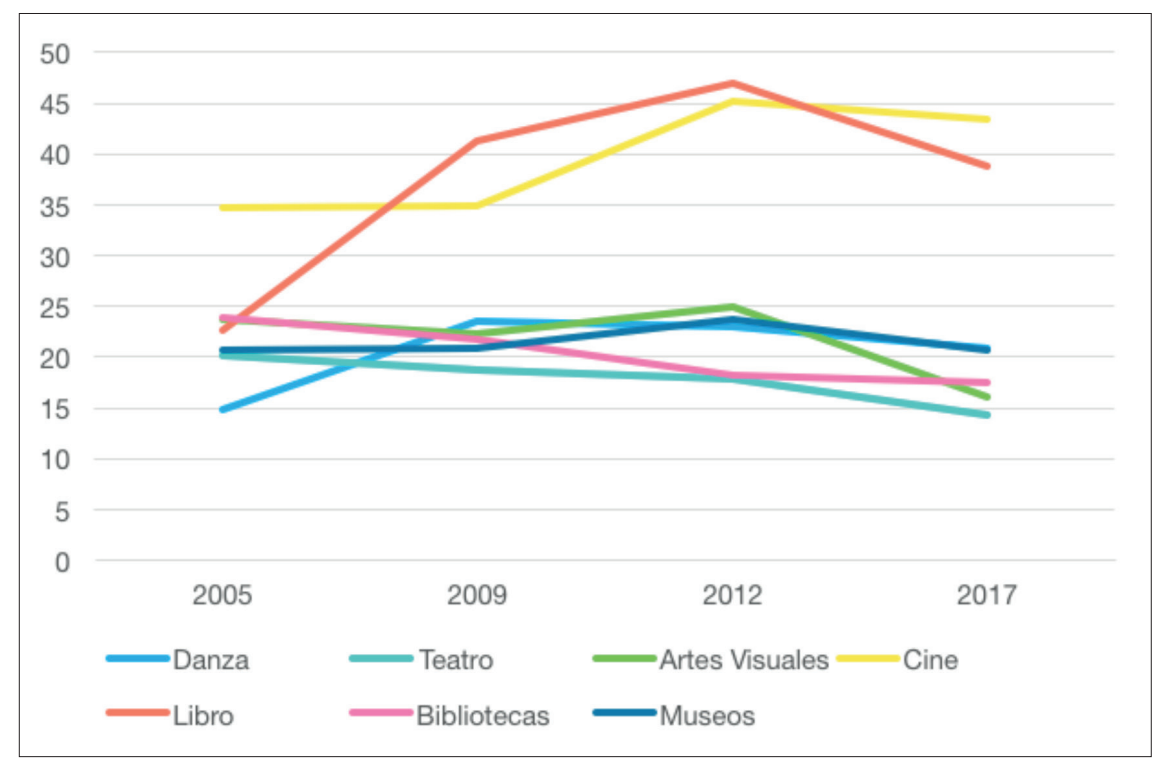

Gráfico 1. Trayectoria Participación Cultural en Chile 2005-2017 (Acceso al menos una vez en los últimos 12 meses). Fuentes: Encuesta de Consumo Cultural, 20032004 (publicada 2005); Segunda Encuesta de Consumo y Participación Cultural, 2009; Tercera Encuesta de Participación y Consumo Cultural 2012; y Cuarta Encuesta de Participación Cultural, 2017. Consejo Nacional de la Cultura y las Artes de Chile, y Ministerio de las Culturas, las Artes y el Patrimonio de Chile.

Desde la medición de 2012 a la de 2017, es posible advertir un decrecimiento en todos los accesos culturales, especialmente a nivel de lectura de libros (con una disminución de $8,1 \%$ entre ambos años) y asistencia a espacios de exhibición de artes visuales (con una diferencia entre ambos años de casi 9\%). En el caso de danza, teatro y museos, las variaciones negativas son de $2 \%, 3,6 \%$ y $3,1 \%$, respectivamente. Los únicos servicios culturales que mantienen una tendencia estable entre ambos años son el cine (diferencia de $1,8 \%)$ y las bibliotecas $(0,8 \%)$.

Como han señalado otros estudios recientes (Pérez, 2018; Palma et al., 2015), los accesos culturales en Chile han exhibido un retroceso importante en los últimos años. Junto con promover discusiones sobre la formulación de políticas culturales y de formación de públicos en contextos de aceleración tecnológica y digitalización de los consumos culturales a nivel global (Stillerman, 2015; Warde, 2017), estas cifras exigen avanzar en nuevas lógicas analíticas e interpretativas a nivel local. 
En efecto, las formas y lógicas del consumo y participación cultural se caracterizan por su plasticidad y permanente variación histórica. Con la reformulación de las lógicas de acceso vía plataformas tecnológicas -por ejemplo, en la literatura (Vlieghe et al., 2016) y los espacios museales (Jafari et al., 2013)-, han emergido en los últimos años a nivel global nuevos grupos de consumidores culturales. Como ha señalado Katz-Gerro et al. (2011), las lógicas de la participación cultural varían en el tiempo y por ende se requiere elaborar nuevas tipologías contextuales (Bergadaà, 2007; Hanquinet, 2013). En el caso chileno la realización de tipologías no ha estado ausente. Torche (2007 y 2010) ha trabajado en profundidad sobre la relación entre estatus social, consumo cultural y prácticas lectoras en diversos perfiles sociales, y Güell et al. (2011) han elaborado, a partir de un análisis factorial y de correspondencias múltiples de la Encuesta de Consumo Cultural 2009, cinco perfiles de consumidores culturales según sus niveles de acceso y frecuencia. Uno de los perfiles identificados fue el de "Consumo cultural alto vía soporte tecnológico". Considerados por aquel entonces como "emergente", este grupo se caracterizó por estar compuesto por jóvenes de nivel socioeconómico alto y medio, y con alta educación. Luego de casi diez años de cambios tecnológicos y una expansión ascendente de posibilidades de acceso a través de dispositivos tecnológicos (celulares, laptops, ipads), ¿qué nuevos perfiles de consumidores culturales han surgido en el Chile actual?, ¿qué nuevas formas de equidad y/o desigualdad cultural han emergido en los últimos años a partir de la expansión tecnológica?

\section{METODOLOGÍA}

A partir de los datos de la "IV Encuesta Nacional de Participación Cultu-

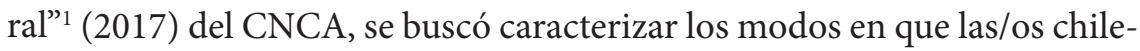
nas/os acceden, se apropian y reconfiguran los bienes y servicios culturales disponibles en la sociedad. En específico, se propuso analizar las modalidades actuales de la participación cultural en Chile. Para ello, y con el fin de definir esos perfiles, se realizó, en primer lugar, un análisis de cluster, utilizando el procedimiento $K$ medias, a partir de las siguientes dimensiones:

- Acceso a bienes y servicios culturales en los últimos 12 meses (Durante los últimos 12 meses, ¿ha ido a alguna presentación o asistido a...?) a

\footnotetext{
${ }^{1}$ La IV Encuesta Nacional de Participación Cultural se aplicó entre agosto y octubre de 2017 y se encuestaron a más de 13 mil personas mayores de 15 años a lo largo del país. El trabajo en terreno fue realizado por el Centro Encuestas y Estudios Longitudinales de la Pontificia Universidad Católica de Chile.
} 
obras de teatro, danza, ópera, conciertos de música clásica y popular, cine, artes visuales, museos, centros culturales y bibliotecas.

- Frecuencia de acceso en los últimos 12 meses: lectura, internet, videojuegos, televisión y radio.

El análisis arrojó una tipología de cuatro grupos. Para chequear la diferenciación entre ellos, se desagregaron, en base a las mismas variables de acceso a bienes y servicios culturales que ingresaron en el proceso de clasificación, por variables sociodemográficas: edad, quintil de ingreso, sexo y nivel educacional. Se realizaron pruebas de chi-cuadrado para testear que haya diferencias estadísticamente significativas entre los grupos que emergen del análisis con respecto a las variables consumo de bienes y servicios culturales, así como respecto de las variables sociodemográficas.

No obstante, la prueba de chi-cuadrado es altamente sensible a los elevados tamaños muestrales, donde es posible sobreestimar como estadísticamente significativas diferencias marginales y precisamente la muestra con que se trabajó el análisis presenta un tamaño elevado (12.151 casos en total, 9.114 clasificados en la tipología). Por ello, las pruebas también fueron aplicadas a una submuestra aleatoria de aproximadamente el 15\% de los casos (1.841 en total, 1.385 clasificados en el cluster) y se observó que la tipología presentó diferencias estadísticamente significativas con respecto a todas las variables de acceso a bienes y servicios culturales.

\section{LAS NUEVAS (O VIEJAS) LÓGICAS DE LA PARTICIPACIÓN CULTURAL EN CHILE}

A partir del análisis realizado, los cuatro grupos creados se caracterizan de la siguiente manera:

1. Grupo 1 (15\%): El 40,7\% tiene entre 15 y 29 años, el 50\% se encuentra entre los dos quintiles más ricos del país, el $20 \%$ posee educación universitaria incompleta (estudiantes principalmente) y un $33,2 \%$ posee una licenciatura o más.

2. Grupo 2 (20\%): el 34,5\% tiene entre 30 y 44 años, casi la mitad se concentra entre los quintiles 3 y 4 de ingreso, y el 36,3\% posee educación media completa. Solo un $15,5 \%$ posee educación universitaria completa.

3. Grupo 3 (37\%): el 47,6\% tiene 60 años o más, el 53\% se ubica en los quintiles más pobres del país y el $17 \%$ posee educación media incompleta (y un $11,1 \%$ educación básica completa). 
4. Grupo 4 (27\%): su rango etario, educación e ingreso es similar a la tipología 2 , aunque se observa mayor porcentaje de mujeres $(58,6 \%)$.

En base a ellos, se procedió a realizar un análisis de correspondencias múltiples que se presenta en el Gráfico 2.

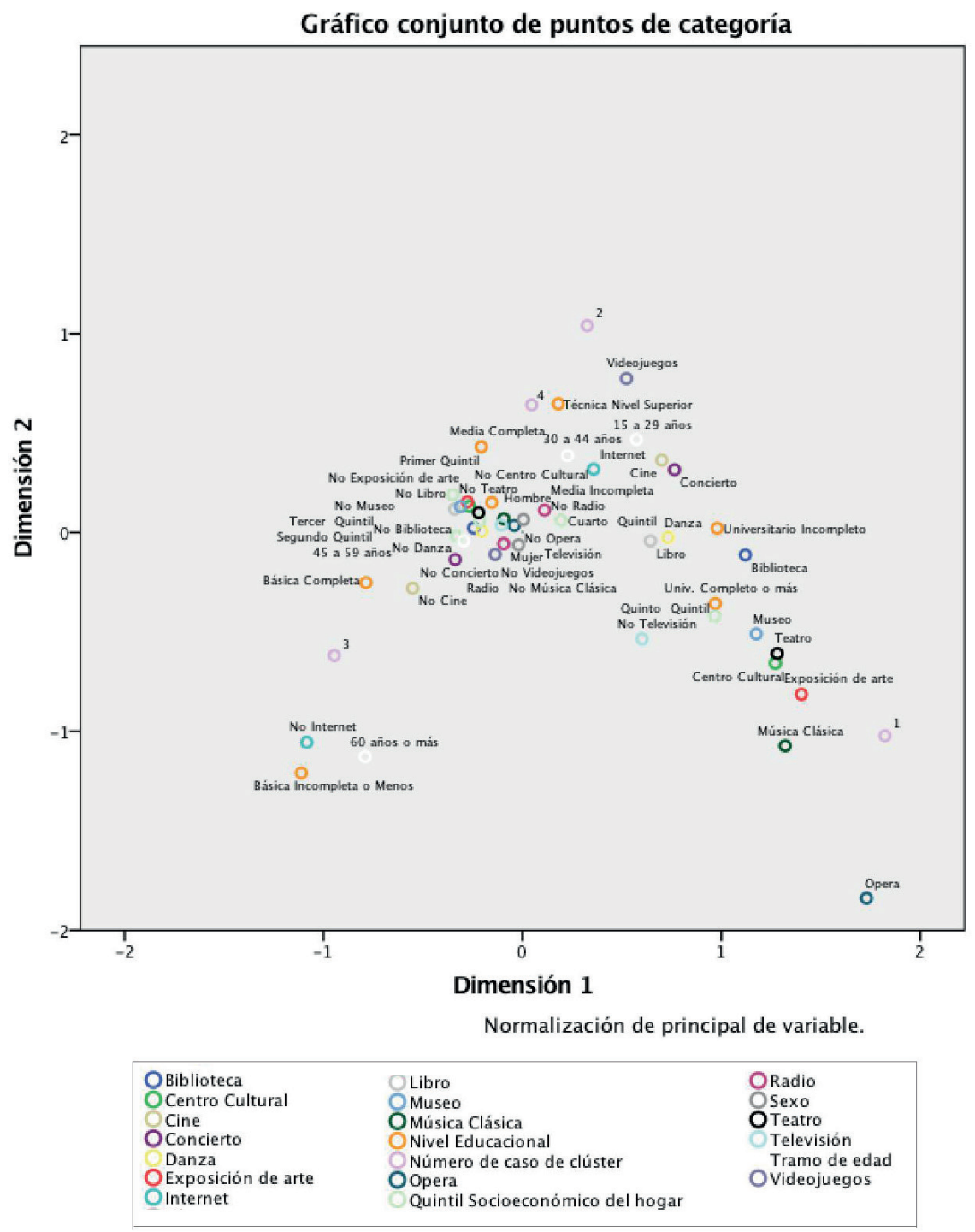

Gráfico 2. Análisis de correspondencias múltiples para cluster de tipología de consumo cultural en Chile. Fuente: Análisis a partir de la Cuarta Encuesta de Participación Cultural, 2017, Ministerio de las Culturas, las Artes y el Patrimonio de Chile. 
En el análisis de correspondencias múltiples se presenta la distribución de los cuatro grupos generados en el análisis de cluster. El grupo 1 se ubica en la sección inferior derecha. Como se observa, los tipos de consumo cultural de este grupo son: ópera, música clásica, exposición de artes visuales, teatro, centro cultural y museo. Además de poseer principalmente educación universitaria completa y pertenecer al quintil más rico de la población, este grupo de "alto consumo cultural" también se caracteriza por tener un porcentaje importante de estudiantes universitarios y jóvenes. El grupo 2 -que corresponde a uno de cada cinco chilenas/os- se ubica en la parte superior derecha del cuadrante. Según los datos, los miembros de este grupo realizan un acceso cultural principalmente de videojuegos, cine y conciertos de música popular. Además de concentrarse en los grupos más jóvenes (entre 15 y 29 años, aunque un porcentaje importante se ubica entre los 30 y 44 años), este grupo exhibe mayores niveles de ingreso económico y escolaridad media. Por su parte, el grupo 3 -que corresponde al $37 \%$ de la población nacional-, se inscribe en la parte inferior izquierda del cuadrante. Este grupo se caracteriza por tener un "consumo cultural muy bajo" a nivel general -su principal acceso es a radio y televisión- y su población está compuesta principalmente por adultos mayores que se ubican en los quintiles más pobres del país. Junto con tener uno de los niveles educativos más bajos, sorprende su especial distancia de Internet. Finalmente, el grupo 4 se ubica en la parte superior izquierda del cuadrante. A diferencia de los grupos 1 y 2, su acceso y consumo cultural es claramente menor: casi no asiste a teatro, danza, exposiciones de artes visuales, museos y bibliotecas. Tampoco lee libros. El único servicio cultural que refleja una inclinación mayor es Internet (dispositivo compartido por el grupo 2). Sumado a ello, y considerando que cerca del $60 \%$ de ese grupo está compuesto por mujeres, son personas de mayor edad, menor educación y menores ingresos. En términos generales, su práctica cultural se asemeja al grupo 3 .

\section{DISCUSIÓN}

El análisis aquí descrito permite realizar ciertas líneas de discusión. En primer lugar, sigue existiendo una diferenciación radical entre quienes realizan un alto nivel de consumo cultural y quienes realizan uno muy bajo. En efecto, al observar por variables sociodemográficas, no hay mayores novedades. Al igual que la evidencia internacional histórica (Chan \& Goldthorpe, 2007; Bennett et al., 2009), en Chile se observa con claridad la relación entre los patrones de consumo y gustos culturales, y la pertenencia de cla- 
se. Los datos han demostrado consistentemente en su historia una fuerte desigualdad de acuerdo con el grupo socioeconómico de pertenencia: es decir, las/os chilenas/os realizan actividades artístico-culturales en base a los capitales económicos y educativos (culturales) que poseen. Esta línea de investigación, que ha sido dirigida en Chile por Modesto Gayo (Gayo et al., 2009 y 2013), pone en cuestionamiento los esfuerzos desarrollados por la institucionalidad cultural por revertir esta situación.

En segundo lugar, resulta interesante constatar que casi la mitad de la población nacional realiza un tipo de consumo cultural que está mediado por Internet y que, cuando se accede a un tipo de servicio cultural que exige un desplazamiento territorial o una disposición avanzada de acceso cultural, es principalmente de asistencia a salas de cine y conciertos de música popular (principalmente de festivales musicales). El resto de los bienes y servicios culturales casi no se integra en sus decisiones culturales. Al mismo tiempo, es interesante observar que un porcentaje importante de la población -grupo 3, correspondiente al 37\% de la población- no tiene acceso al uso de las redes tecnológicas o no las utiliza para acceder a la oferta cultural disponible.

En tercer lugar, y al revisar estudios previos realizados en Chile con datos estadísticos de hace diez años (Güell et al., 2011), se ha generado, aparentemente, una agrupación o integración entre los grupos que Güell et al. identificaron de "Consumo alto vía soporte tecnológico", "Consumo medio" y "Consumo medio-bajo" en dos grupos (identificados en este estudio como grupo 2 y 4). Esta constatación, que resulta de una interpretación aún especulativa, permite establecer, por una parte, una nueva arista analítica sobre cómo las tecnologías están siendo apropiadas por la población joven y adulto-joven y, por otra, nos exige pensar las consecuencias que esto puede tener en el uso de los espacios artístico-culturales tradicionales. En el primer caso, las tecnologías se han inscrito en la vida cotidiana de una población importante del país. Según la OECD (2021), en los últimos años el acceso a Internet en los hogares en Chile ha crecido exponencialmente (un 87,5\% de los hogares cuenta con acceso a la red) y, gracias a ello, se podría suponer una ampliación de las posibilidades -potenciales- de acceso cultural. Con la disponibilidad creciente en todos los niveles socioeconómicos de dispositivos tecnológicos en el hogar y personales (telefonía móvil), es posible desprender la emergencia de nuevas formas de consumo cultural en música (Spotify), videos musicales o presentaciones de teatro y danza vía online (YouTube), películas (sitios por streaming o Netflix), lectura (periódicos online, textos en redes sociales, noticias compartidas) y obras de artes visuales (Pinterest, Instagram), entre otras. Estas nuevas formas de acceso cultural 
ponen en tensión las lógicas tradicionales de asistencia a los espacios artísticos tradicionales. Como se evidenció en el análisis histórico de los consumos culturales en Chile, la tendencia señala una disminución al acceso a la oferta cultural que exige un consumo cultural situado (Campos, 2012).

\section{CONCLUSIONES}

Las tecnologías de la información están cambiando las lógicas históricas del consumo cultural. Hoy, gracias a una ampliación acelerada del consumo debido a los mercados de créditos -así como también por las nuevas tecnologías de la información-, el acceso cultural se ha vuelto cada vez más complejo y enmarcado en lógicas globales. En otros términos, lo que históricamente entendimos por "consumo cultural" no ha disminuido, sino que ha cambiado en sus formas de apropiación y uso (García Canclini, 2015).

Los resultados de este estudio ofrecen insumos analíticos relevantes para pensar las políticas culturales en países como Chile. Como se ha señalado, en los últimos años los bienes culturales han adquirido cada vez mayor importancia política y sociológica a raíz de la masificación del consumo de las clases medias y bajas, por el peso creciente de los mercados en la vida cotidiana, por la discursividad política de los derechos culturales y sociales, y por la importancia de los intercambios de símbolos en las redes globales de la industria creativa y tecnológica. Teóricamente, estos procesos -sumado a la precarización transversal de la vida cotidiana (trabajo, pensiones, servicios públicos, relaciones afectivas, etc.)- han generado una aceleración y complejización significativa tanto en las identidades personales de los chilenos como en las dinámicas de sus prácticas culturales.

Bajo este escenario, y según la tipología aquí descrita, las prácticas de consumo y participación cultural no solo mantienen una fuerte correlación con nivel socioeconómico y capital cultural -una vieja constatación que se vuelve a reforzar-, sino que también se mantienen diferencias significativas en las prácticas culturales entre cada grupo (especialmente en la tipología 1 y 3). Ciertamente, las tecnologías han ingresado en la vida cotidiana de gran parte de la población adulto-joven del país. Pero, como queda en evidencia, esto no ha implicado un aumento en las lógicas de acceso a espacios artístico-culturales tradicionales (teatro, danza, artes visuales, museos, bibliotecas, etcétera), sino que, más bien, ha producido, al parecer, un tipo de ciudadano que concentra su consumo cultural a través de Internet y en festivales de música popular y acceso a salas de cine. A pesar de todos los esfuerzos institucionales y políticos por aumentar los accesos culturales 
(tradicionales), no se han observado diferencias significativas a nivel poblacional durante los últimos quince años.

Lo anterior nos exige pensar las políticas culturales en Chile desde nuevos marcos de análisis. En un contexto donde la precarización general de la vida cotidiana en aspectos como la jubilación, el trabajo, la educación, el transporte y la salud se acrecienta, entonces se deben desplegar intervenciones públicas que privilegien otras dimensiones socioculturales antes que las eminentemente artísticas. Per Mangset (2020) se sitúa en ese registro de problemas y plantea que, en las últimas décadas, se ha hecho evidente una crisis de la noción histórica de políticas culturales. Su diagnóstico apunta a una serie de elementos complementarios para el estudio aquí desarrollado: la democratización cultural no ha dado respuestas concretas al acceso a las artes, sino que ha profundizado ciertas lógicas de desigualdad cultural tales como la centralización de la oferta cultural y la escasa inversión cultural en zonas históricamente deprivadas; a pesar de los esfuerzos de museos y espacios culturales por aumentar sus públicos y desarrollar estrategias de vinculación territorial, estos siguen siendo lugares considerados obsoletos que refuerzan barreras tanto económicas como simbólicas en sus ciudadanos; los y las trabajadoras/os culturales siguen teniendo ingresos económicos bajos e inestables, así como también escasa previsión social y seguros de salud; la excesiva burocratización y concursabilidad de las políticas culturales ha generado una "jaula de hierro" en las dinámicas culturales y creativas de las/os artistas, priorizando recursos a las/os artistas con mayores niveles de capital cultural y social; y, en los últimos años, se ha evidenciado el estancamiento y/o reducción -e incluso cierre de Ministerios- de los presupuestos estatales en materia cultural. Todos estos aspectos exigen establecer un nuevo orden de discusiones teórico-políticas sobre la intervención estatal en materia cultural. En otros términos, en un mundo caracterizado por la hiperconectividad, los algoritmos y el Bigdata, resulta cada vez más difícil hacer frente a los desafíos actuales del acceso cultural. Según las estadísticas culturales de países como España (Ariño y Llopis, 2017), Argentina (SINCA, 2017), Brasil (Machado et al., 2017) y, como aquí lo hemos demostrado, en el caso chileno, las personas asisten cada vez menos a bibliotecas, museos, centros culturales, salas de concierto, teatros, salas de danza y galerías de artes visuales. Este fenómeno en curso tiene implicancias directas en la forma de pensar el consumo cultural en América Latina (Wortman y Radakovich, 2019), así como también en el detrimento del uso de los espacios culturales, ya que estos son promotores de "esferas públicas deliberativas" (Barrett, 2012) y no son simplemente lugares de exhibición. 
En definitiva, y considerando el contexto de la pandemia acaecida durante el año 2020, la pregunta desplegada en este estudio se vuelve aún más crítica. A raíz de ella, se ha develado y radicalizado el diagnóstico aquí esbozado: la precariedad de las/os trabajadores culturales se ha vuelto crítica, las políticas culturales desde la institucionalidad cultural no han dado respuesta a las demandas del campo artístico y los públicos han migrado hacia plataformas digitales. Si comprendemos el acceso a la cultura como un derecho humano (Bennoune, 2019), entonces no solo se necesitan nuevas estrategias críticas para pensar las futuras -y potenciales- formas de desigualdad cultural, sino también los esfuerzos necesarios para revertir las débiles condiciones estructurales tanto de las/os artistas como de los espacios de circulación cultural en Chile.

\section{REFERENCIAS}

Antoine, C. (2009). Audiencias y consumo cultural en Chile: ¿Tópico o justificación de un modelo de democratización de la cultura: 1990-2005? RePresentaciones: Periodismo, Comunicación y Sociedad, 5, 65-83.

Ariño, A. y Llopis, R. (2017). Culturas en tránsito. Las prácticas culturales en España en el comienzo del siglo XXI. Madrid: Fundación SGAE.

Barrett, J. (2012). Museums and the Public Sphere. West Sussex: Wiley-Blackwell. Bennett, T., Savage, M., Silva, E., Warde, A., Gayo, M., \& Wright, D. (2009). Culture, Class, Distinction. London: Routledge.

Bennoune, K. (2019). Informe de la Relatora Especial sobre los derechos culturales. Naciones Unidas. Recuperado de https://undocs.org/es/A/74/255

Bergadaà, M. (2007). Temporal Frameworks and Individual Cultural Activities. Four typical profiles. Time \& Society 66(2-3), 387-408.

Brunner, J.J. (1990). Políticas culturales: apuntes a partir del caso chileno. $\mathrm{Pa}$ pers. Revista de Sociología 35, 117-132.

Brunner, J.J. (2005). Chile: Ecología social del cambio cultural. En C. Catalán, y P. Torche (eds.). Consumo cultural en Chile. Miradas y perspectivas. Santiago: INE-CNCA-La Nación.

Campos, L. (2012). El consumo cultural: una actividad situada. En P. Güell y T. Peters (eds.). La trama social de las prácticas culturales. Sociedad y subjetividad en el consumo cultural de los chilenos. Santiago: Ediciones Universidad Alberto Hurtado.

Carrasco, E. y Negrón, B. (eds.) (2005). Industrias culturales: un aporte al desarrollo. Santiago: Lom.

Carrasco, E. y Negrón, B. (2006). La cultura durante el período de transición a la democracia. 1990-2006. Santiago: Ediciones del Consejo Nacional de la Cultura y las Artes. 
Catalán, C. y Sunkel, G. (1990). Consumo cultural en Chile. La élite, lo masivo y lo popular. Santiago: FLACSO.

Chan, T.W. \& Goldthorpe, J. (2007). Social stratification of cultural consumption across three domains: Music, Theatre, Dance and Cinema, and the Visual Arts. En T. Chan (ed.). Social Status and Cultural Consumption. Cambridge: Cambridge University Press.

CNCA (2014). Prácticas de consumo, participación y valoración de la cultura en Chile: Etnografía de análisis de casos. Santiago: Consejo Nacional de la Cultura y las Artes.

CNCA (2017). Catastro de infraestructura cultural Pública y Privada. Santiago: Consejo Nacional de la Cultura y las Artes.

De Cea, M. (2010). Expert knowledge mediation in the relationship between cultural stakeholders, politics and the state. International Journal of Politics, Culture and Society 23(4), 191-200.

De Cea, M. (2017). El sendero de la institucionalidad cultural chilena: cambios y continuidades. Estudios Públicos 145, 103-132.

García Canclini, N. (ed.) (2015). Hacia una antropología de los lectores. México D.F.: Ariel.

Gayo, M., Teitelboim, B. y Méndez, M. (2009). Patrones culturales de uso del tiempo libre en Chile. Una aproximación desde la teoría bourdieuana. Universum 24(2), 42-72.

Gayo, M., Teitelboim, B. y Méndez, M. (2013). Exclusividad y fragmentación: los perfiles culturales de la clase media en Chile. Universum 28(1), 97-128.

Garretón, M.A., (2008). Las políticas culturales en los gobiernos democráticos en Chile. En A. Albino y R. Bayardo (eds.). Políticas culturais na Ibero-América. Salvador, Brasil: EDUFBA.

Güell, P. (2012). Las políticas culturales son prácticas sociales: discusiones sobre sus consecuencias metodológicas. En B. Negrón y M. I. Silva (eds.). Políticas Culturales: ¿Qué medimos? ¿Cómo evaluamos? Santiago: Lom.

Güell, P., Peters, T. y Morales, R. (2011). Tipología de prácticas de consumo cultural en Chile a inicios del siglo XXI: mismas desigualdades, prácticas emergentes, nuevos desafíos. Universum 2(26), 121-141.

Hanquinet, L. (2013). Visitors to modern and contemporary art museums: towards a new sociology of 'cultural profiles'. The Sociological Review 61, 790-813.

Jafari, A, Taheri, B., \& Vom Lehn, D. (2013). Cultural consumption, interactive sociality, and the museum. Journal of Marketing Management 29(15-16), 1729-1752.

Katz-Gerro, T., Halkier, B., \& Martens, L. (2011). Applying practice theory to the study of consumption: Theoretical and methodological considerations. Journal of Consumer Culture 11(1), 3-13.

Machado, A., Golgher, A., Diniz, S., \& Gama, L. (2017). Consumption of cultural goods and services and time allocation in Brazil. Nova Economia 27(1), 35-63. https://doi.org/10.1590/0103-6351/3115 
Mangset, P. (2020). The end of cultural policy? International Journal of Cultural Policy 26(3), 398-411. DOI: 10.1080/10286632.2018.1500560

Martineau, J. (2017). Culture in the age of acceleration, hypermodernity, and globalized temporalities. The Journal of Arts Management, Law, and Society 47(4), 218-229.

Miller, T. (2018). El trabajo cultural. Barcelona: Gedisa.

Muñoz, N. (2011). La reforma cultural en Chile: un ejemplo de polarización en la gestión de una política pública. Revista Chilena de Administración Pública 18, 61-91.

Navarro, A. (2006). Cultura: ¿quién paga? Gestión, infraestructura y audiencias en el modelo chileno de desarrollo cultural. Santiago: Ril.

Odello, M. (2011). The right to take part to cultural life: General comment No21 of the United Nations Committee on Economic, Social and Cultural Rights. Anuario Español de Derecho Internacional 27, 493-521.

OECD (2021). Internet access (indicator). doi: 10.1787/69c2b997-en. Recuperado de https://www.oecd-ilibrary.org/science-and-technology/internetaccess/indicator/english_69c2b997-en

PNUD. (2002). Informe de Desarrollo Humano: Nosotros los chilenos, un desafío cultural. Santiago: PNUD.

Pérez, C. (2018). La participación cultural. Análisis del discurso de la política cultural chilena sobre teatro. Política y Cultura 49, 9-27.

Palma, T., Alvarado, P., y García, I. (2015). Audiencias y estrategias de convocatoria en festivales de cine nacional. Comunicación y Medios 30, 255-270. DOI:10.5354/0719-1529.2015.31610

Palominos, S. (2018). Paradigms of participation in the National Council for Culture and Arts: challenges on representation, recognition, access to creation and reception in post-dictatorship Chilean public cultural policy. International Journal of Cultural Policy 24(2), 164-185. DOI: $10.1080 / 10286632.2016 .1156099$

Pinochet, C. (2016). Abrir las grandes alamedas. Festivales culturales y espacio público en la construcción de un imaginario de la democracia. Revista Estudios Avanzados 26, 1-18.

Rampaphorn, N. (ed.) (2008). Ciudadanía, participación y cultura. Santiago: Lom. SINCA (2017). Encuesta Nacional de Consumos Culturales 2017. Buenos Aires: Sistema de Información Cultural de la Argentina.

Stillerman, J. (2015). The sociology of consumption. A global approach. Cambridge: Polity Press.

Subercaseux, B. (2016). Políticas culturales en Chile: una perspectiva histórica. Estudios Públicos 144, 205-232.

Torche, F. (2007). Social status and cultural consumption: The case of reading in Chile. Poetics, 35, 70-92.

Torche, F. (2010) Social status and public cultural consumption: Chile in comparative perspective. En T. Chan (ed.). Social Status and Cultural Consumption. Cambridge: Cambridge University Press. 
Ubilla, L. (2010). Identidades juveniles en Mala Onda de Alberto Fuguet: la colonización del imaginario político por el consumo. Chile, 1980-1990. Revista de Humanidades, 22, 141-164.

Vlieghe, J., Muls, J., \& Rutten, K. (2016). Everybody reads: reader engagement with literature in social media environments. Poetics, 54, 25-37.

Warde, A. (2017). Consumption. A Sociological Analysis. London: PalgraveMacmillan.

Wortman, A. y Radakovich, R. (eds.) (2019). Mutaciones del consumo cultural en el siglo XXI: tecnologías, espacios y experiencias. Buenos Aires: Teseo. 